\title{
1 Influenza H3 and H1 hemagglutinins have different genetic barriers for resistance to broadly neutralizing stem antibodies
}

4 Nicholas C. Wu ${ }^{1, *}$, Andrew J. Thompson ${ }^{2, *}$, Juhye M. Lee ${ }^{3,4,5}$, Wen Su ${ }^{6}$, Britni M. Arlian ${ }^{2}$, Jia $^{2}$ $\mathrm{Xie}^{7}$, Richard A. Lerner ${ }^{7,8}$, Hui-Ling Yen ${ }^{6}$, Jesse D. Bloom ${ }^{3,4,9}$, lan A. Wilson ${ }^{1,8, \S}$

$7 \quad{ }^{1}$ Department of Integrative Structural and Computational Biology, The Scripps Research

8 Institute, La Jolla, CA 92037, USA

$9 \quad{ }^{2}$ Department of Molecular Medicine, The Scripps Research Institute, La Jolla, CA 92037, USA

$10{ }^{3}$ Basic Sciences Division, Fred Hutchinson Cancer Research Center, Seattle, WA 98109, USA

$11{ }^{4}$ Department of Genome Sciences, University of Washington, Seattle, WA 98195, USA

$12{ }^{5}$ Medical Scientist Training Program, University of Washington, Seattle, WA 98195, USA

$13{ }^{6}$ School of Public Health, Li Ka Shing Faculty of Medicine, The University of Hong Kong, Hong

14 Kong SAR, China

$15{ }^{7}$ Department of Chemistry, The Scripps Research Institute, La Jolla, CA, 92037, USA

$16{ }^{8}$ The Skaggs Institute for Chemical Biology, The Scripps Research Institute, La Jolla, CA

17 92037, USA

$18{ }^{9}$ Howard Hughes Medical Institute, Fred Hutchinson Cancer Research Center, Seattle, WA

1998109, USA

20 * These authors contributed equally to this work

$21 \S$ To whom correspondence may be addressed. Email: wilson@scripps.edu (I.A.W.) 


\section{ABSTRACT}

23 In the past decade, the discovery and characterization of broadly neutralizing antibodies

24 (bnAbs) to the highly conserved stem region of influenza hemagglutinin (HA) have provided

25 valuable insights for development of a universal influenza vaccine. However, the genetic barrier

26 for resistance to stem bnAbs has not been thoroughly evaluated. Here, we performed a series

27 of deep mutational scanning experiments to probe for resistance mutations. We found that the

28 genetic barrier to resistance to stem bnAbs is generally very low for the $\mathrm{H} 3$ subtype but

29 substantially higher for the $\mathrm{H} 1$ subtype. Several resistance mutations in $\mathrm{H} 3$ cannot be

30 neutralized by stem bnAbs at the highest concentration tested, do not reduce in vitro viral fitness

31 and in vivo pathogenicity, and are often present in circulating strains as minor variants. Thus,

$32 \mathrm{H} 3 \mathrm{HAs}$ have a higher propensity than $\mathrm{H} 1 \mathrm{HAs}$ to escape major stem bnAbs and creates a

33 potential challenge in the development of a bona fide universal influenza vaccine.

\section{ONE SENTENCE SUMMARY}

36 Acquisition of resistance by influenza virus to broadly neutralizing hemagglutinin stem

37 antibodies varies tremendously depending on subtype. 


\section{Introduction}

39 The major surface antigen of influenza virus, the hemagglutinin (HA), is composed of a highly

40 variable globular head domain that houses the receptor binding site and a conserved stem

41 domain that is responsible for membrane fusion (1). All of the major antigenic sites on HA are

42 located on the HA globular head (2-5), which is immunodominant over the stem (6). However,

43 most antibodies to the globular head domain are strain-specific. In contrast, although harder to

44 elicit during natural infection or vaccination, many HA stem antibodies have impressive cross-

45 reactive breadth $(7,8)$. The isolation, characterization and structure determination of broadly

46 neutralizing antibodies (bnAbs) to the HA stem over the past decade have provided tremendous

47 insights into antiviral and vaccine development against influenza virus (9), including immunogen

48 design towards a universal influenza vaccine (10-12). Several stem bnAbs are also currently in

49 clinical trials as therapeutics (13). Stem bnAbs have also provided templates for design of small

50 proteins, peptides and small molecules against influenza virus (14-18). Therefore, while

51 influenza virus remains a major global health concern, stem bnAbs open up multiple promising

52 avenues to tackle this challenging problem.

54 However, emergence of resistance mutations can be a major obstacle for antiviral and vaccine

55 development. Several studies have reported difficulty in selecting strong resistance mutations to

56 stem bnAbs even after extensive passaging of the viruses (19-21), or through deep mutational

57 scanning (22), which is a comprehensive and unbiased approach (23). Nonetheless, strong

58 resistance mutations have been reported in other studies through virus passaging $(20,24,25)$.

59 It is unclear then why some studies were able to identify strong resistance mutations while

60 others could not. Here we systematically compare how readily resistance can emerge to stem

61 bnAbs in $\mathrm{H} 3$ and $\mathrm{H} 1 \mathrm{HAs}$, and find that there are major differences between the subtypes.

62

\section{Deep mutational scanning of the major HA stem epitope}


64 CR9114 (26) and Fl6v3 (27) are two bnAbs that bind the HA stem and have exceptional

65 neutralization breadth. They are in fact two of the known bnAbs with the greatest breadth

66 against influenza viruses. Both Fl6v3 and CR9114 neutralize group 1 and 2 influenza A viruses

67 (26, 27), and CR9114 further cross-reacts with influenza B HA (26). Deep mutational scanning

68 (23), which combines saturation mutagenesis and next-generation sequencing, has previously

69 been applied to study how HA mutations affect influenza viral fitness (28-30), and to identify

70 viral mutants that are resistant to anti-HA antibodies (31). Here, we employed deep mutational

71 scanning of the HA stem on influenza virus to search for resistance mutations to CR9114 and

72 Fl6v3. We focused on eight HA2 residues in the HA stem of H3N2 A/Hong Kong/1/1968

73 (H3/HK68): namely Q42, 145, D46, Q47, 148, N49, L52, and T111 (Fig. 1, A to C). All except

74 T111 are located on HA2 helix A, which is a common target for stem bnAbs. Residues 42,45 ,

$7546,48,49,52$ interact with CR9114 and FI6v3, whereas residue 47 only interacts with FI6v3

76 (Fig. 1, D and E). The completely buried T111 was also selected because its mutation in H5 HA

77 enabled escape from CR6261 (24), which binds a similar epitope to CR9114 (26, 32).

79 We quantified the in vitro fitness of 147 out of 152 possible single viral mutants and 6,234 out of

8010,108 possible double viral mutants across the eight residues of interest in H3/HK68 HA2

81 under five different conditions: no antibody, $2 \mu \mathrm{g} / \mathrm{mL}$ CR9114 lgG, $10 \mu \mathrm{g} / \mathrm{mL}$ CR9114 lgG, 0.3

$82 \mu \mathrm{g} / \mathrm{mL}$ Fl6v3 IgG, and $2.5 \mu \mathrm{g} / \mathrm{mL}$ FI6v3 IgG (fig. S1). In the absence of antibody, many viral

83 mutants have a relative fitness [proxy for replication fitness (30)], similar to wild type (WT),

84 which was set as 1 (Fig. 2, A and B), and indicate that the HA stem region can tolerate many

85 mutations.

86

87 We further quantified the relative resistance of each viral mutant by normalizing their relative

88 fitness in the presence and absence of antibody (fig. S2). Many resistance mutations to CR9114

89 and Fl6v3 were observed (Fig. 2, C and D) and most were located at HA2 residues 42 and 45, 
90 which form an important component of the binding interface with CR9114 and FI6v3 (Fig. 1, D

91 and $\mathrm{E})$. In addition, the double mutants also showed high relative resistance if one mutation

92 exhibited high relative resistance even if the other did not (fig. S3). Overall, these results

93 demonstrate the prevalence of $\mathrm{H} 3 / \mathrm{HK} 68$ resistance mutations to stem bnAbs and mutations

94 with cross-resistance to both CR9114 and Fl6v3, even though these bnAbs are encoded by

95 different germline genes and have very different angles of approach to the HA (8).

97 Validation of resistance mutations

98 To validate our findings from deep mutational scanning, 24 single and double HA mutants of the

$99 \mathrm{H} 3 / \mathrm{HK} 68$ virus that spanned a range of relative resistance were individually constructed and

100 tested against CR9114 and FI6v3 IgGs (Fig. 3A). The minimum inhibitory concentration (MIC) in

101 a microneutralization assay strongly correlated with the relative resistance from deep mutational

102 scanning (Spearman's rank correlation > 0.8, Fig. 3B) with several viral mutants showing strong

103 cross-resistance to both CR9114 and FI6v3. For example, the MICs of CR9114 and FI6v3

104 against mutants $145 \mathrm{Y} / \mathrm{S} / \mathrm{N} / \mathrm{F} / \mathrm{W}$ were all $>100$ and $\geq 20 \mu \mathrm{g} \mathrm{mL} \mathrm{m}^{-1}$, respectively, compared to 3.1

105 and $0.2 \mu \mathrm{g} \mathrm{mL}^{-1}$ for WT. This validation experiment substantiates our finding that strong

106 resistance mutations are prevalent in $\mathrm{H} 3 / \mathrm{HK} 68$.

\section{Natural occurrence of resistance mutations}

109 Next, we explored whether these resistance mutations were found in naturally circulating strains

110 While most strong resistance mutations have not yet been observed in naturally circulating

111 strains, it is important to note that a few could be identified at low frequency in natural human

$112 \mathrm{H} 3 \mathrm{~N} 2$ isolates (33), including 145T, I45M and N49D (Fig. 4A). I45T is also observed in human

$113 \mathrm{H} 3 \mathrm{~N} 2$ isolates sequenced without any passaging (fig. S4A), implying that its presence was not

114 due to a passaging artifact (34). Moreover, the strong cross-resistance mutation I45F was found

115 in all human H2N2 viruses that circulated from 1957 to 1968 (Fig. 4B, fig. S4B), while almost all 
116 avian $\mathrm{H} 2 \mathrm{~N} 2$ viruses have lle45 (Fig. 4C), and explains why it is more difficult for human H2N2

117 viruses to be bound or neutralized by some stem bnAbs compared to other subtypes $(24,26$,

118 35). Thus, these findings suggest that some resistance mutations to stem bnAbs already occur

119 in circulating strains.

120

\section{In vivo pathogenicity and escape of resistance mutations}

122 We further tested the in vivo viral pathogenicity of HA2 mutants $145 \mathrm{~T}, 145 \mathrm{M}$, and $145 \mathrm{~F}$, which are

123 of relevance to circulating strains (see above). The weight loss profiles in mice after infection by

124 HA2 mutant and WT viruses were comparable (fig. S5), indicating that these resistance

125 mutations do not reduce in vivo pathogenicity. We further demonstrated that 145T, 145M, and

126 I45F were able to escape in vivo prophylactic protection. While mice infected with WT were

127 completely protected by CR9114 $\mathrm{lgG}$ at all tested doses $\left(1,4\right.$, and $\left.10 \mathrm{mg} \mathrm{kg}^{-1}\right)$, mutants I45T,

128 I45M, and I45F were lethal even at the highest dose of CR9114 IgG (Fig. 4, D to G).

\section{Resistance mutations decrease affinity to bnAbs}

131 To dissect the resistance mechanism, we tested the binding of H3/HK68 I45T, I45M, and I45F

132 recombinant HAs to CR9114 and FI6v3, and also to another stem bnAb $27 \mathrm{F3}$ (35), which

133 utilizes the same $V_{H} 1-69$ germline as CR9114 and similarily neutralizes group 1 and 2 influenza

134 A viruses. The binding $\left(\mathrm{K}_{\mathrm{d}}\right)$ of CR9114 Fab, CR9114 IgG, $27 \mathrm{~F} 3 \mathrm{Fab}, 27 \mathrm{~F} 3 \mathrm{lg}$, and FI6v3 lgG

135 was all diminished against the HA mutants compared to WT (Table 1 and fig. S6), and was

136 particularly dramatic with the I45F mutant, where binding was undetectable to CR9114 Fab and

$137 \mathrm{IgG}$, and 27F3 Fab and IgG. In contrast, the binding of these stem Fabs and IgGs to N49T,

138 which did not exhibit any resistance against CR9114 and FI6v3 (Fig. 2C, Fig. 3A), are

139 comparable to the WT (Table 1). As a control, we also tested binding of bnAb S139/1 that

140 targets the receptor-binding site far from the stem epitope (36, 37). S139/1 IgG affinities against 
141 those HA mutants $\left(\mathrm{K}_{d}=1.8 \mathrm{nM}\right.$ to $\left.3.1 \mathrm{nM}\right)$ were similar to WT $\left(\mathrm{K}_{d}=2.1 \mathrm{nM}\right)$. Thus, virus

142 resistance to stem bnAbs correlated with a decrease in binding affinity to the mutant HAs.

144 To understand the structural basis of the resistance, we determined crystal structures of HAs

145 with HA2 mutations I45T, I45M, and I45F to 2.1 to $2.5 \AA$ resolutions (table S1 and fig. S7A).

146 Compared to WT (lle45), the shorter side chain of I45T would create a void when CR9114 is

147 bound (fig. S7B) that would be energetically unfavorable. In contrast, the longer flexible side

148 chain of I45M would likely clash with CR9114 (fig. S7B), but CR9114 is still able to bind the

149 I45M mutant, albeit with much lower affinity than WT (Table 1). The I45F mutant, however,

150 makes a more severe clash with CR9114 and no binding was detected (Table 1, fig. S7B).

151 Similar observations for Fl6v3 (fig. S7C) explain the sensitivity of CR9114 and Fl6v3 to

152 mutations at $\mathrm{HA} 2$ residue 45.

153

\section{Resistance to HA stem bnAbs is subtype specific}

155 We further aimed to examine whether those mutations that confered strong resistance in

$156 \mathrm{H} 3 / \mathrm{HK} 68$ would have the same phenotypes in other H3 strains. Consequently, we examined the

157 phenotype of three resistance mutations of relevance to circulating strains (see below), namely

158 I45T, I45M, and I45F, on an H3N2 A/Wuhan/359/95 (H3/Wuhan95) genetic background. These

159 three viral mutants had WT-like titer after viral rescue and passaging (fig. S8A), showed similar

160 plaque size as WT (fig. S8B), and conferred strong resistance to FI6v3 (fig. S8, C and D).

161 Therefore, these mutants have similar phenotypes in H3/HK68 and H3/Wuhan95 viruses. This

162 result led us to hypothesize that strong resistance mutants to stem bnAbs are readily attainable

163 in a wide range of $\mathrm{H} 3$ strains, as well as to explore whether the same phenomenon can be

164 observed in $\mathrm{H} 1$ subtype, which is the other currently circulating influenza $A$ subtype in the 165 human population. 
167 Previously, two of the authors here performed deep mutational scanning to search for 168 resistance mutants of $\mathrm{H} 1 \mathrm{~N} 1 \mathrm{~A} / \mathrm{WSN} / 33$ (H1/WSN) virus to FI6v3 (22). In contrast to this study, 169 resistance mutations to Fl6v3 were rare in H1/WSN, and had only very small effects. This 170 discrepancy suggested that the prevalence of resistance mutations is markedly different

171 between $\mathrm{H} 3 / \mathrm{HK} 68$ and H1/WSN. We therefore performed four additional deep mutational

172 scanning experiments - three with H1N1 strains, namely A/Solomon Islands/3/2006 (H1/SI06)

173 against FI6v3, A/Michigan/45/2015 (H1/Mich15) against Fl6v3, H1/WSN against CR9114, and

174 one with H3N2 strain A/Perth/16/2009 (H3/Perth09) against Fl6v3. The H1/SI06 and H1/Mich15

175 HA mutant virus libraries contain all possible single amino-acid substitutions at HA2 residues

$17642,45,46,47,48,49,52$, and 111, whereas H1/WSN and H3/Perth09 HA mutant virus libraries

177 both contain all possible single substitutions across the entire HA and were constructed in

178 previous studies $(38,39)$. We also analyzed the previously published dataset on H1/WSN

179 against Fl6v3 (22).

180

181 To compare H1/SI06, H1/Mich15, H1/WSN and H3/Perth09 to H3/HK68, we computed the

182 relative resistance of mutations at $\mathrm{HA} 2$ residues $42,45,46,47,48,49,52$, and 111 (Fig. 5, A to

183 E). Similar to H3/HK68, resistance mutations are highly prevalent in H3/Perth09 (Fig. 5C).

184 Conversely, resistance mutations were rare in H1/SI06 (Fig. 5A), H1/Mich15 (Fig. 5B), and

185 H1/WSN (Fig. 5, D and E). We further calculated the fraction surviving (22) for each viral mutant

186 across the entire H1/WSN and H3/Perth09 HA proteins during antibody selection. Fraction

187 surviving is a quantitative measure for the resistance that is normalized across deep mutational

188 scanning experiments (22). The fraction surviving values of H1/WSN mutants against CR9114

189 were all very small, similar to previous observations of H1/WSN against FI6v3 (Fig. 5F, fig.

190 S10). In stark contrast, many mutants of H3/Perth09 were identified with a large fraction

191 surviving value (Fig. 5F). Consistent with the relative resistance profile of H3/HK68 (Fig. 2C), a

192 number of H3/Perth09 mutants with a large fraction surviving value were again at HA2 residues 
19342 and 45 (Fig. 5F and fig. S11A). Moreover, mutations at HA2 residue 53, which were not

194 examined in H3/HK68 (Fig. 2), had high fraction surviving in H3/Perth09 against Fl6v3 (fig. S11,

$195 \mathrm{~A}$ and $\mathrm{B})$. In $\mathrm{H} 3 \mathrm{HA}$, mutation of $\mathrm{HA} 2$ residue 53 would abolish a hydrogen bond to the

196 complementarity-determining region (CDR) H3 of Fl6v3 (fig. S11B). Together, these results

197 suggest that the prevalence of resistance mutations to stem bnAbs is a general phenomenon for

198 the H3 subtype, but not the H1 subtype.

200 Subtype-specific differences in the HA stem

201 We next aimed to elucidate the mechanism that underlies the lower genetic barrier to resistance

202 to stem bnAbs in $\mathrm{H} 3 \mathrm{HA}$ as compared to $\mathrm{H} 1 \mathrm{HA}$. Many mutations at HA2 residue 45 have a high

203 fitness cost in $\mathrm{H} 1 / \mathrm{SI} 06$ (fig. S9A), which can increase the genetic barrier to resistance. However,

204 most mutations at HA2 residue 45 have no fitness cost in $\mathrm{H} 1 /$ Mich15. In addition, the mutational

205 fitness profiles of $\mathrm{H} 1 / \mathrm{SI} 06$ and $\mathrm{H} 1 /$ Mich15 (fig. S9, A and B) show that many mutations can be

206 tolerated in the HA stem, similar to H3/HK68 (Fig. 2A, fig. S9, C to F). Thus, the difference in

207 genetic barrier to resistance to stem bnAbs between $\mathrm{H} 1$ and $\mathrm{H} 3$ subtypes cannot be fully

208 explained by their ability to tolerate mutations (i.e. fitness cost of mutations).

210 We therefore further compared the structures of CR9114 in complex with H3 HA and in complex

211 with H5 HA (Fig. 5G) (26). Since the structure of CR9114 with H1 HA is not available, CR9114

212 with H5 HA was used instead, as it also belongs to group $1 \mathrm{HAs}$ and is therefore more similar to

$213 \mathrm{H} 1$ than to H3 HA (group 2). Structural comparison indicates that CR9114 packs tighter to the

214 helix A of H3 HA than to H5 HA. Specifically, there is $\sim 1 \AA$ difference in the position of the Ca of

215 HA2 Ile45. Subsequently, a bulkier substitution at HA2 Ile45, such as I45M, would create a

216 larger disruption of the CR9114-HA binding interface in the context of H3 subtype. Thus, subtle

217 differences in the binding of bnAbs to different HA subtypes may lead to differences in how 218 antibodies are affected by mutations in or near the epitope. 
220 Similar observations can be made for FI6v3. The orientation of Tyr100c on CDR H3 of FI6v3

221 differs when binding to $\mathrm{H} 1$ or $\mathrm{H} 3 \mathrm{HAs}$ (Fig. $5 \mathrm{H})$ (27). The position of HA2 lle45 also differs

222 between $\mathrm{H} 1$ and $\mathrm{H} 3 \mathrm{HAs}$ when Fl6v3 is bound. As a result, Tyr100c of Fl6v3 packs tighter to

223 HA2 Ile45 of H3 than to H1 HA. Thus, a bulkier substitution at HA2 lle45 will disrupt binding

224 between FI6v3 and H3 HA to a greater extent than Fl6v3 to H1 HA. Therefore, the low genetic

225 barrier to resistance to stem bnAbs in the $\mathrm{H} 3$ subtype can be at least partly attributed to both

226 high mutational tolerance in the HA stem and subtype-specific structural features. While a

227 number of subtype-specific structural features are known in the stem region (40), how these

228 structural differences influence the genetic barriers for resistance to stem bnAbs remains to be

229 addressed in future studies.

\section{Ramifications for escape from a universal vaccine or therapeutic stem bnAbs}

232 Prior studies of influenza bnAbs have not considered whether different subtypes might have

233 different abilities to generate resistance mutations against bnAbs. A major finding here is that

$234 \mathrm{H} 3 \mathrm{HA}$ has a much lower genetic barrier to resistance to two of the broadest bnAbs, CR9114

235 and Fl6v3, as compared to $\mathrm{H} 1 \mathrm{HA}$. This observation is consistent with the literature, where

236 strong resistance to other human HA stem antibodies have been reported in H3 subtype (20,

$23725,41)$ versus none $(20)$ to weak resistance $(21,22)$ in the $\mathrm{H} 1$ subtype. Therefore, it may be

238 easier for stem bnAbs to maintain suppression of the $\mathrm{H} 1$ subtype than the $\mathrm{H} 3$ subtype.

240 Since the HA stem is immunosubdominant to the globular head domain, immunological

241 pressure on the HA stem may not have been sufficient to impact the evolution of circulating

242 influenza strains (42). However, several stem bnAbs are currently in clinical trials for therapeutic

243 purposes (13) and a number of recently developed influenza vaccine immunogens have

244 focused on targeting the HA stem $(8,9)$. If stem bnAbs begin to be distributed on a global scale, 
245 the immunological pressure on the HA stem will certainly surge to a level not previously seen.

246 Our findings here indicate that resistance mutations could emerge, at least in $\mathrm{H} 3$ subtype.

248 Although resistance mutations to stem bnAbs are still rare in currently circulating influenza

249 strains (Fig. 4A), it is important to evaluate the potential impact of such mutations since many

250 vaccine strategies aim to elicit anti-stem antibodies. In fact, we were not able to overcome some

251 key resistance mutations (I45T, I45M, and I45F) by in vitro evolution of CR9114 (fig. S12).

252 Nonetheless, the best strategy to prevent or overcome such resistance may involve delivery or

253 elicitation of a combination of antibodies with different resistance profiles. In addition, it remains

254 to be explored whether stem bnAbs exist or can be generated that are difficult to escape from

255 the H3 subtype. The discovery and characterization of bnAbs with different escape profiles will

256 therefore continue to be key to broaden our arsenal against influenza virus. For example,

257 human $\mathrm{H} 2 \mathrm{~N} 2$ virus, which carries a Phe at HA2 residue 45, often has low reactivity with stem

258 bnAbs $(24,26,27,35,43)$, although a very few can have high potency against human H2N2

259 (44-46). Future studies on anti-stem responses against human H2N2 and emerging viruses,

260 such as $\mathrm{H} 5 \mathrm{~N} 1$ and $\mathrm{H} 7 \mathrm{~N} 9$, may provide further insights into how to overcome potential

261 resistance when immune pressure is transferred to the HA stem. 


\section{REFERENCES AND NOTES}

263 1. I. A. Wilson, J. J. Skehel, D. C. Wiley, Structure of the haemagglutinin membrane

264 glycoprotein of influenza virus at $3 \AA$ resolution. Nature 289, 366-373 (1981).

2. D. C. Wiley, I. A. Wilson, J. J. Skehel, Structural identification of the antibody-binding sites of Hong Kong influenza haemagglutinin and their involvement in antigenic variation. Nature 289, 373-378 (1981).

3. J. J. Skehel et al., A carbohydrate side chain on hemagglutinins of Hong Kong influenza viruses inhibits recognition by a monoclonal antibody. Proc Natl Acad Sci U S A 81, 1779-1783 (1984).

271 4. A. J. Caton, G. G. Brownlee, J. W. Yewdell, W. Gerhard, The antigenic structure of the

272 influenza virus A/PR/8/34 hemagglutinin (H1 subtype). Cell 31, 417-427 (1982).

273 5. W. Gerhard, J. Yewdell, M. E. Frankel, R. Webster, Antigenic structure of influenza virus

6. F. Krammer, P. Palese, Advances in the development of influenza virus vaccines. Nat Rev Drug Discov 14, 167-182 (2015).

8. N. C. Wu, I. A. Wilson, A perspective on the structural and functional constraints for immune evasion: insights from influenza virus. J Mol Biol 429, 2694-2709 (2017).

10. A. Impagliazzo et al., A stable trimeric influenza hemagglutinin stem as a broadly

11. H. M. Yassine et al., Hemagglutinin-stem nanoparticles generate heterosubtypic

12. S. A. Valkenburg et al., Stalking influenza by vaccination with pre-fusion headless HA

13. E. Sparrow, M. Friede, M. Sheikh, S. Torvaldsen, A. T. Newall, Passive immunization for influenza through antibody therapies, a review of the pipeline, challenges and potential applications. Vaccine 34, 5442-5448 (2016).

292 14. S. J. Fleishman et al., Computational design of proteins targeting the conserved stem 
16. A. Chevalier et al., Massively parallel de novo protein design for targeted therapeutics. Nature 550, 74-79 (2017).

298 17. R. U. Kadam et al., Potent peptidic fusion inhibitors of influenza virus. Science 358, 496$299502(2017)$.

300 18. M. J. P. van Dongen et al., A small-molecule fusion inhibitor of influenza virus is orally active in mice. Science 363, eaar6221 (2019).

302 19. G. Nakamura et al., An in vivo human-plasmablast enrichment technique allows rapid identification of therapeutic influenza A antibodies. Cell Host Microbe 14, 93-103 (2013).

$30420 . \quad$ N. Chai et al., Two escape mechanisms of influenza A virus to a broadly neutralizing stalk-binding antibody. PLoS Pathog 12, e1005702 (2016).

21. C. S. Anderson et al., Natural and directed antigenic drift of the $\mathrm{H} 1$ influenza virus hemagglutinin stalk domain. Sci Rep 7, 14614 (2017).

22. M. B. Doud, J. M. Lee, J. D. Bloom, How single mutations affect viral escape from broad and narrow antibodies to $\mathrm{H} 1$ influenza hemagglutinin. Nat Commun 9, 1386 (2018).

23. D. M. Fowler, S. Fields, Deep mutational scanning: a new style of protein science. Nat Methods 11, 801-807 (2014).

24. M. Throsby et al., Heterosubtypic neutralizing monoclonal antibodies cross-protective against $\mathrm{H} 5 \mathrm{~N} 1$ and $\mathrm{H} 1 \mathrm{~N} 1$ recovered from human $\operatorname{lgM}^{+}$memory B cells. PLoS One 3, e3942 (2008).

25. R. H. Friesen et al., A common solution to group 2 influenza virus neutralization. Proc Natl Acad Sci U S A 111, 445-450 (2014).

26. C. Dreyfus et al., Highly conserved protective epitopes on influenza B viruses. Science 337, 1343-1348 (2012).

27. D. Corti et al., A neutralizing antibody selected from plasma cells that binds to group 1 and group 2 influenza A hemagglutinins. Science 333, 850-856 (2011).

321 28. N. C. Wu et al., High-throughput profiling of influenza A virus hemagglutinin gene at single-nucleotide resolution. Sci Rep 4, 4942 (2014).

29. B. Thyagarajan, J. D. Bloom, The inherent mutational tolerance and antigenic evolvability of influenza hemagglutinin. eLife 3, e03300 (2014).

30. N. C. Wu et al., Diversity of functionally permissive sequences in the receptor-binding site of influenza hemagglutinin. Cell Host Microbe 21, 742-753 (2017). 
33. R. B. Squires et al., Influenza research database: an integrated bioinformatics resource for influenza research and surveillance. Influenza Other Respir Viruses 6, 404-416 (2012).

34. N. C. Wu et al., A structural explanation for the low effectiveness of the seasonal influenza H3N2 vaccine. PLoS Pathog 13, e1006682 (2017).

35. S. Lang et al., Antibody $27 \mathrm{~F} 3$ broadly targets influenza A group 1 and 2 hemagglutinins

36. R. Yoshida et al., Cross-protective potential of a novel monoclonal antibody directed through a further variation in $\mathrm{V}_{\mathrm{H}} 1-69$ antibody orientation on the HA stem. Cell Rep 20, 2935-2943 (2017).

37. P. S. Lee et al., Heterosubtypic antibody recognition of the influenza virus hemagglutinin receptor binding site enhanced by avidity. Proc Natl Acad Sci U S A 109, 17040-17045 (2012). against antigenic site B of the hemagglutinin of influenza A viruses. PLoS Pathog 5 , e1000350 (2009).

38. M. B. Doud, J. D. Bloom, Accurate measurement of the effects of all amino-acid mutations on influenza hemagglutinin. Viruses 8, E155 (2016).

39. J. M. Lee et al., Deep mutational scanning of hemagglutinin helps predict evolutionary fates of human H3N2 influenza variants. Proc Natl Acad Sci U S A 115, E8276-E8285 (2018).

40. S. J. Zost, N. C. Wu, S. E. Hensley, I. A. Wilson, Immunodominance and antigenic variation of influenza virus hemagglutinin: implications for design of universal vaccine immunogens. J Infect Dis 219, S38-S45 (2019).

41. D. C. Ekiert et al., A highly conserved neutralizing epitope on group 2 influenza A viruses. Science 333, 843-850 (2011).

42. E. Kirkpatrick, X. Qiu, P. C. Wilson, J. Bahl, F. Krammer, The influenza virus hemagglutinin head evolves faster than the stalk domain. Sci Rep 8, 10432 (2018).

43. M. G. Joyce et al., Vaccine-induced antibodies that neutralize group 1 and group 2 influenza A viruses. Cell 166, 609-623 (2016).

44. Y. Okuno, Y. Isegawa, F. Sasao, S. Ueda, A common neutralizing epitope conserved between the hemagglutinins of influenza A virus $\mathrm{H} 1$ and $\mathrm{H} 2$ strains. $J$ Viro/ 67, 25522558 (1993).

45. C. Dreyfus, D. C. Ekiert, I. A. Wilson, Structure of a classical broadly neutralizing stem antibody in complex with a pandemic $\mathrm{H} 2$ influenza virus hemagglutinin. $J$ Virol 87, 71497154 (2013).

46. N. L. Kallewaard et al., Structure and function analysis of an antibody recognizing all influenza A subtypes. Cell 166, 596-608 (2016). 


\section{ACKNOWLEDGEMENTS}

369 We thank Wenli Yu and Geramie Grande for technical support in protein expression, Steven

370 Head, Jessica Ledesma and Padmaja Natarajan at TSRI Next Generation Sequencing Core

371 and Fred Hutch Genomics Core for next-generation sequencing, Matthew Haynes and Brian

372 Seegers of the TSRI Flow Cytometry Core Facility for performing FACS, Alfred Ho, Eva-Maria

373 Strauch, and Barney Graham for insightful discussions, and James Paulson for his continual

374 support. Funding: We acknowledge support from the Bill and Melinda Gates Foundation

375 OPP1170236 (to I.A.W.), NIH K99 Al139445 (to N.C.W.), F30 Al136326 (to J.M.L.), R01

376 Al127893 (to J.D.B.), R56 Al127371 (to I.A.W.), and R01 Al114730 (to J.C.P.). J.M.L. was

377 supported in part by the Center for Inference and Dynamics of Infectious Diseases (CIDID),

378 funded by NIH U54 GM111274. J.D.B. is an Investigator of the Howard Hughes Medical

379 Institute. Author Contributions: N.C.W., J.M.L., R.A.L., H.L.Y., J.D.B., and I.A.W. conceived

380 and designed the study. N.C.W. and J.M.L. performed the deep mutational scanning

381 experiments. N.C.W., J.M.L., and J.D.B. performed the computational data analysis. N.C.W.

382 performed the structural analysis and yeast display experiment. N.C.W. and W.S. performed

383 functional characterization of the viral mutants. A.J.T. and B.M.A. performed the in vivo

384 characterization of the viral mutants. N.C.W. and J.X. produced the CR9114 and $27 \mathrm{F3}$

385 antibodies. J.M.L. produced the Fl6v3 antibody. N.C.W. and I.A.W. wrote the paper and all

386 authors reviewed and edited the paper. Competing interests: The authors declare no

387 competing interests. Data and materials availability: Raw sequencing data will be deposited to

388 the NIH Short Read Archive prior to publication. The X-ray coordinates and structure factors will

389 be deposited to the RCSB Protein Data Bank prior to publication. Links to computer codes and

390 processed data are in supplementary materials. All the other data that support the conclusions

391 of the study are available from the corresponding author upon request.

392

393 SUPPLEMENTARY MATERIALS 
bioRxiv preprint doi: https://doi.org/10.1101/2019.12.30.891135; this version posted December 30, 2019. The copyright holder for this preprint (which was not certified by peer review) is the author/funder, who has granted bioRxiv a license to display the preprint in perpetuity. It is made available under aCC-BY 4.0 International license.

\section{Materials and Methods}

395 Figs. S1 to S12

396 Tables S1 to S3

397 References 47-66 
Fig. 1

A

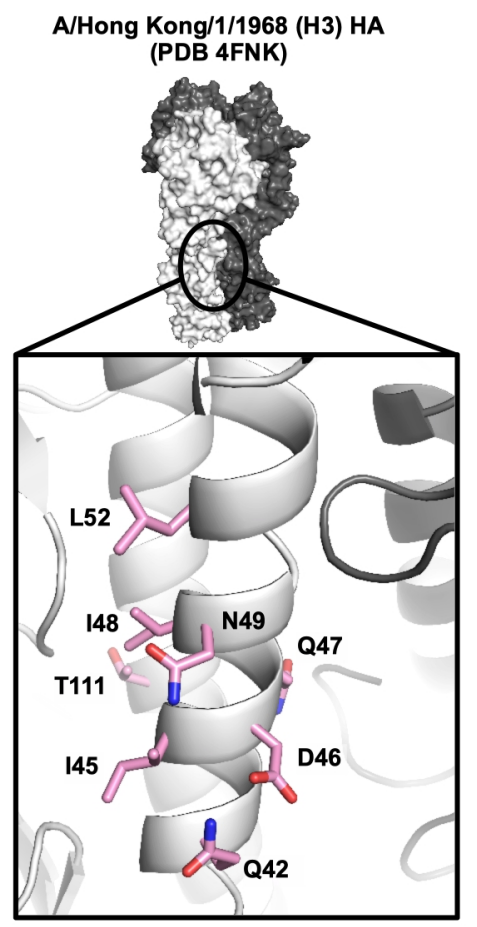

B

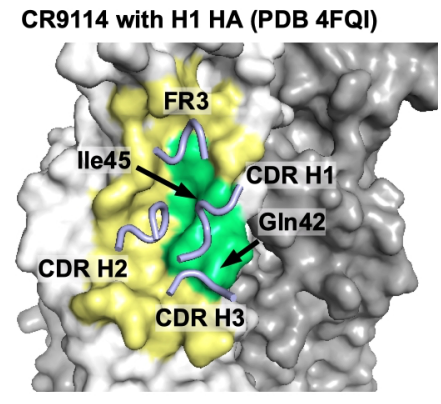

C

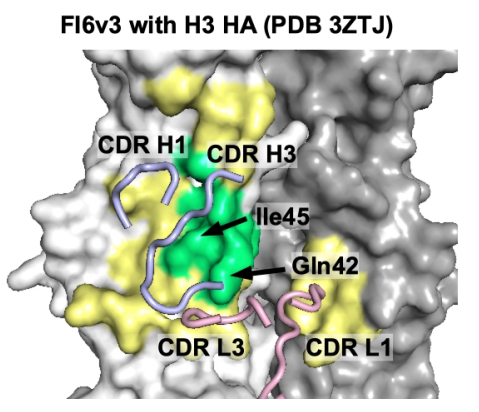

D

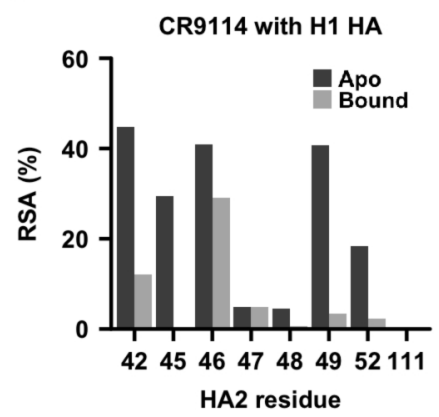

E

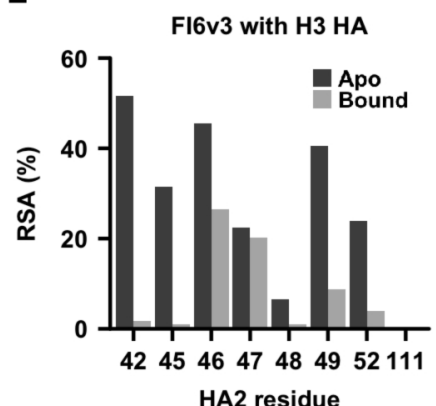

401 Fig. 1. Epitopes of broadly neutralizing antibodies to the HA stem. (A) The location of

402 residues of interest in this study on the HA structures. All residues of interest are on HA2. One

403 protomer of the trimer is shown in light gray and the other two protomers in dark gray and a

404 detailed view of the location of the residues of interest is shown in the inset. (B-C) Epitopes of

405 (B) CR9114 Fab in complex with H1 HA (PDB 4FQI) (26) and (C) FI6v3 in complex with H3 HA

406 (PDB 3ZTJ) (27) are colored in yellow and green, and residues of interest colored in green. The

407 arrows indicate the positions of $\mathrm{HA} 2$ residues 42 and 45 , which are in the center of the bnAb

408 epitopes. Antibody paratopes (CDRs and FR regions) are shown in tube representation and

409 labeled accordingly. Blue: heavy chain. Pink: light chain. (D-E) The relative solvent accessibility

410 (RSA) of each residue of interest is shown. Black bar: apo form. Gray bar: Fab-bound form. 
Fig. 2

A

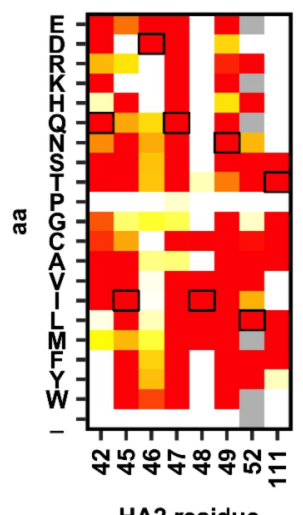

B

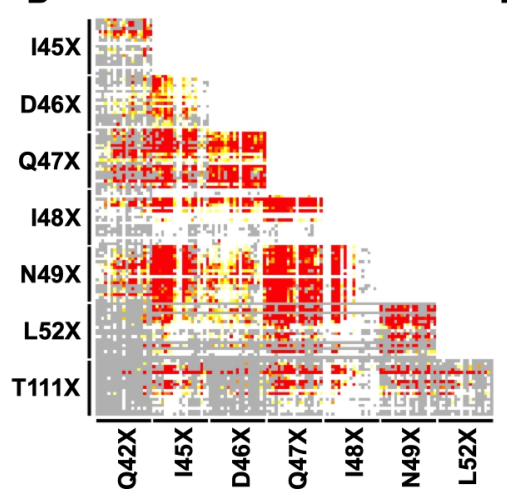

C

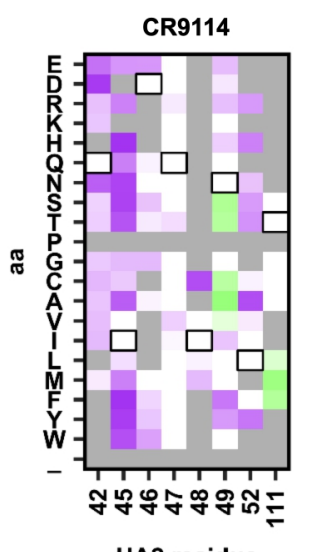

HA2 residue

D

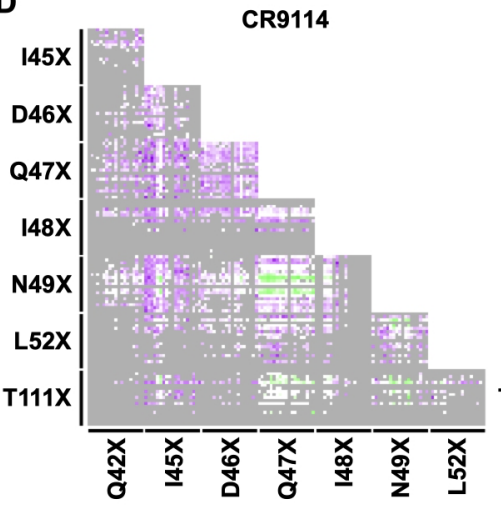

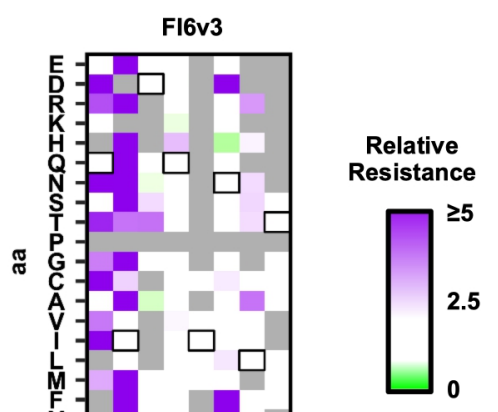

w.

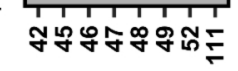

HA2 residue

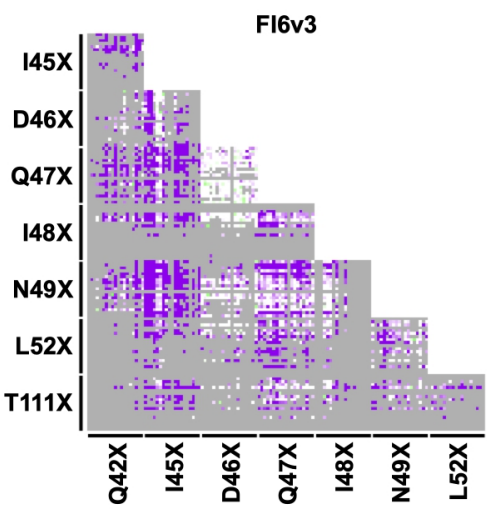

413 Fig. 2. Fitness and resistance profile of H3/HK68 HA2 single and double viral mutants. (A-

414 B) Based on the deep mutational scanning experiment, the relative fitness of $(A)$ single and (B)

415 double mutants are shown with wild type (WT) set as 1. In (A) and (B), mutants with a next-

416 generation sequencing read count of less than 20 from the plasmid mutant library are excluded

417 and shown as grey. (C-D) Relative resistance for (C) each single and (D) each double mutant

418 against $10 \mu \mathrm{g} / \mathrm{mL}$ CR9114 antibody or $2.5 \mu \mathrm{g} / \mathrm{mL}$ Fl6v3 antibody is shown. Relative resistance

419 for WT is set as 1 . In (C) and (D), mutants with a relative fitness of less than 0.5 are shown as

420 grey. Residues correspond to WT sequence are boxed. In (B) and (D), each color point in the

421 heatmap represents a double mutant. Of note, some mutants with an increased sensitivity to

422 antibody are shown in green on the relative resistance color scale. 
Fig. 3

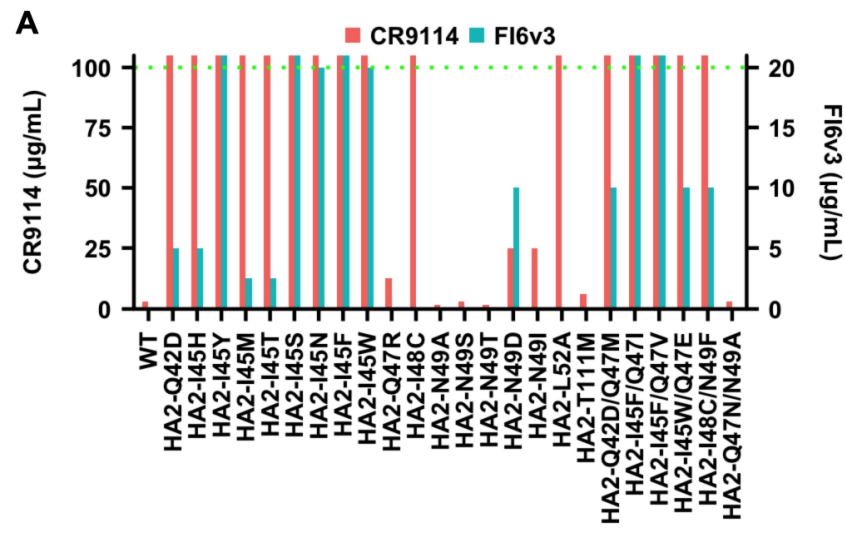

B
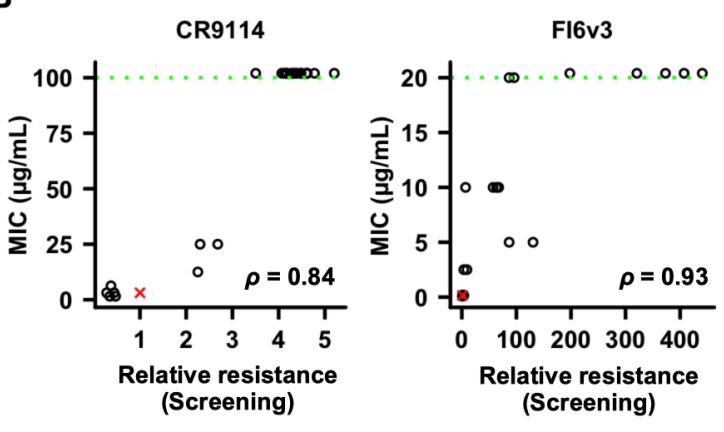

425 Fig. 3. Characterization of antibody-resistant mutants. (A) The minimum inhibitory

426 concentration (MIC) of CR9114 and Fl6v3 to individual viral mutants are shown. The MIC of

427 CR9114 is in red and represented by the y-axis on the left. The MIC of FI6v3 is in blue and

428 represented by the $y$-axis on the right. (B) The Spearman's rank correlations $(\rho)$ between the

429 MIC measured from individual mutants and the relative resistance (against $10 \mu \mathrm{g} / \mathrm{mL}$ CR9114

430 antibody or $2.5 \mu \mathrm{g} / \mathrm{mL}$ Fl6v3 antibody) computed from the profiling experiment (screening) are

431 shown. The green dashed line represents the upper detection limit in A and $\mathbf{B}$. Wild type is

432 represented by the red " $X$ ". 
Fig. 4

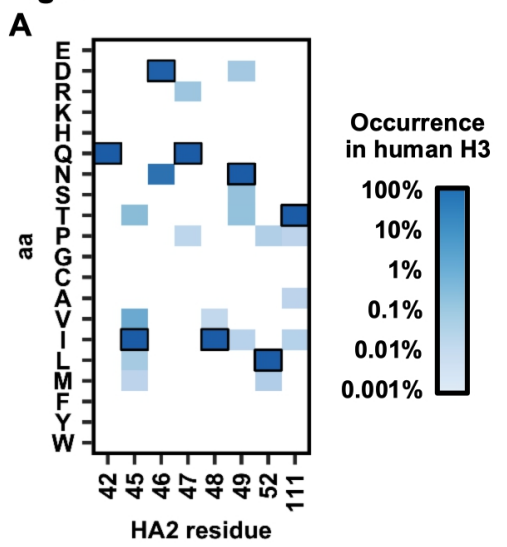

B

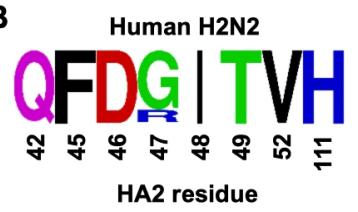

C

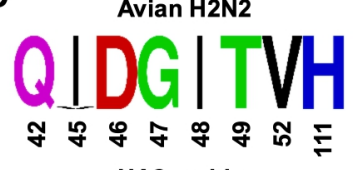

D
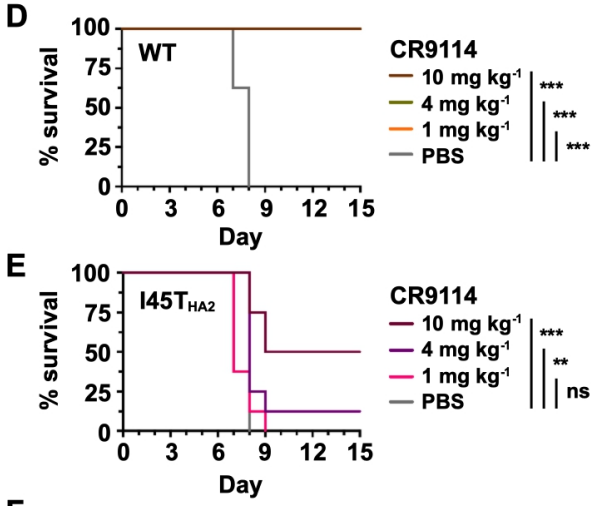

$\mathbf{F}$

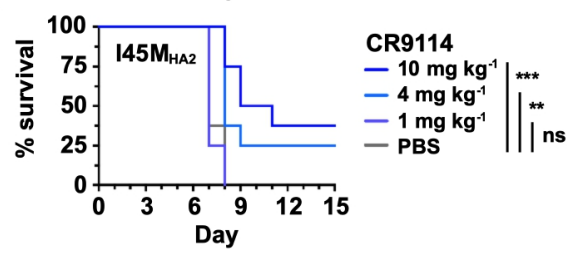

G

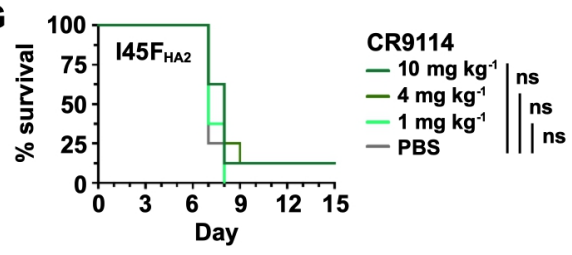

435 Fig. 4. In vivo characterization and natural occurrence of antibody-resistant mutants. (A)

436 The natural occurrence frequencies of different amino-acid variants at the residues of interest in

437 human $\mathrm{H} 3 \mathrm{HAs}$ are shown as a heatmap. (B-C) The natural occurrence frequencies of different

438 amino-acid variants at the residues of interest in (B) human H2N2 HA or (C) avian H2N2 HA are

439 shown as sequence logos. (D-G) Prophylactic protection experiments were performed with

440 different doses of CR9114 against (D) WT, (E) HA2 I45T mutant, (F) HA2 I45M mutant, and (G)

441 HA2 I45F mutant. Recombinant H3/HK68 (7:1 on H1/PR8 backbone) viruses were used. Lethal

442 doses $\left(25 \mathrm{mLD}_{50}\right)$ of WT or mutant viruses were used. Kaplan-Meier survival curves are shown.

443 Paired analysis of each treatment group, relative to control, was conducted using Log-rank

444 (Mantel-Cox) tests. ${ }^{* * *}$ indicates $p$-value $\leq 0.001 ;{ }^{* *}$ indicates $p$-value $\leq 0.01$; * indicates $p$-value

$445 \leq 0.05 ;$ ns (not significant) indicates p-value $>0.05$ 
Fig. 5
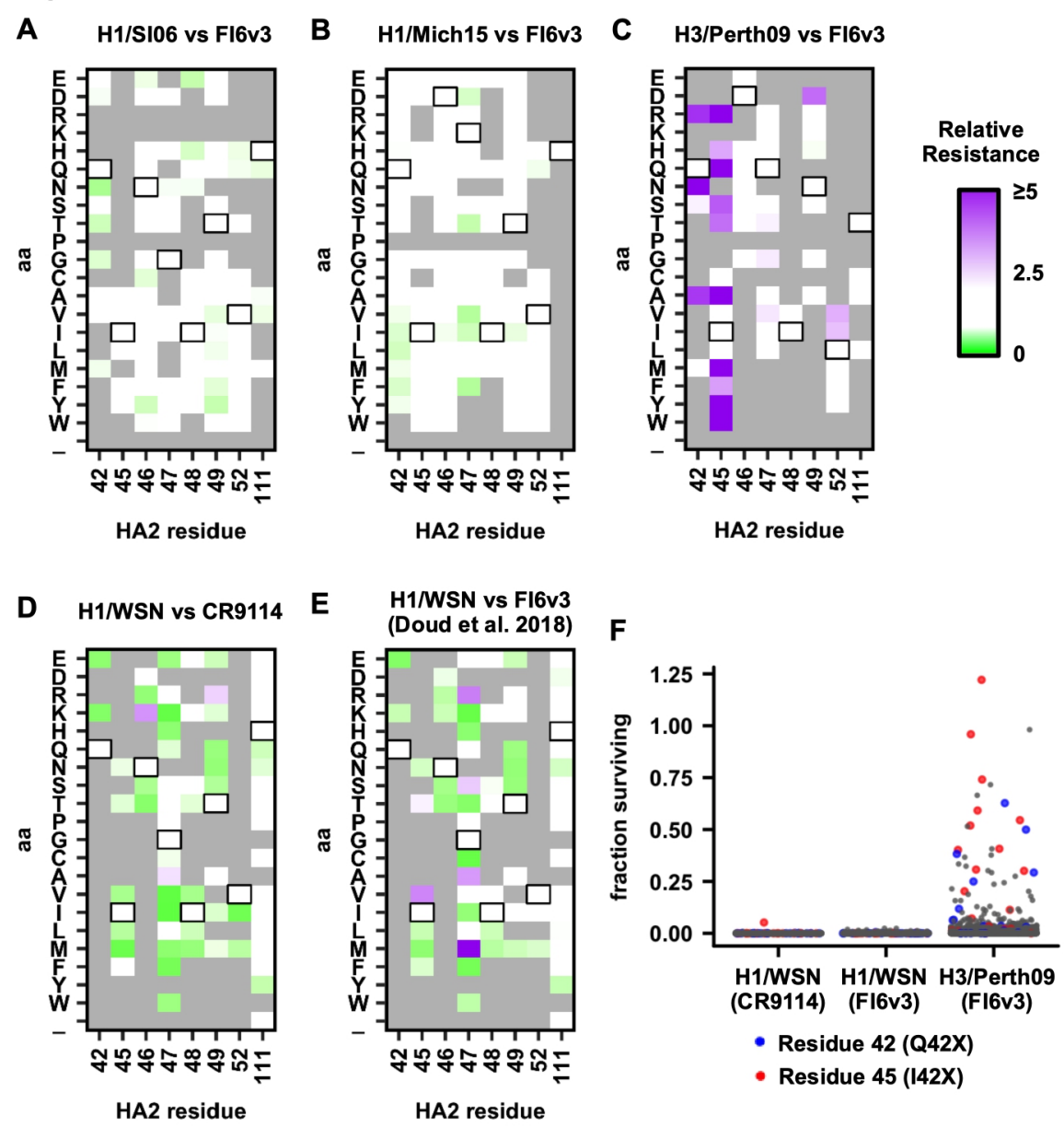

G

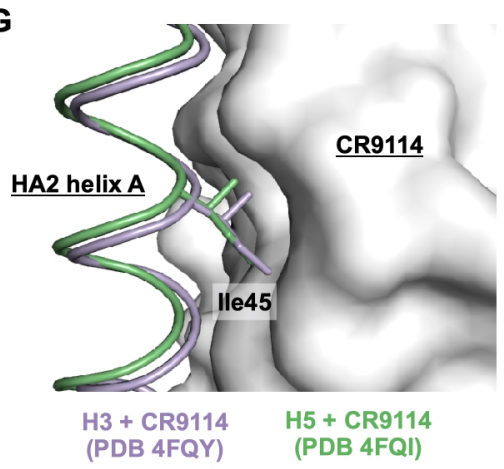

- Residue 42 (Q42X)

- Residue 45 (142X)

H

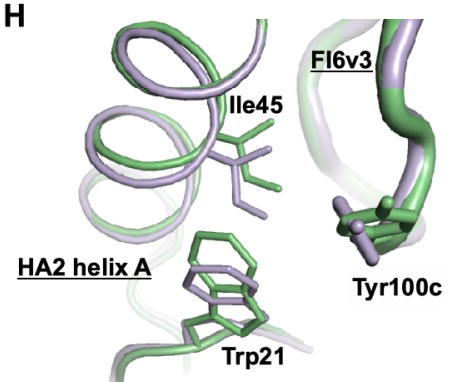

$\begin{array}{cc}\mathrm{H} 3+\mathrm{FI} / 6 \mathrm{v} 3 & \mathrm{H} 1+\mathrm{Fl} \mathrm{vv} 3 \\ (\mathrm{PDB} 3 \mathrm{ZTJ}) & (\mathrm{PDB} 3 \mathrm{ZTN})\end{array}$

448 Fig. 5. Relative resistance profile of single mutants in multiple strains of influenza virus.

449 (A-E) Relative resistance is measured for each single viral mutant at HA2 residues 42, 45, 46,

$45027,48,49,52$, and 111 of (A) H1/SI06 against 300 ng/mL FI6v3 antibody, (B) H1/Mich15

451 against $300 \mathrm{ng} / \mathrm{mL}$ Fl6v3 antibody, (C) H1/WSN against $100 \mathrm{ng} / \mathrm{mL}$ CR9114 antibody, (D)

452 H1/WSN against $200 \mathrm{ng} / \mathrm{mL}$ Fl6v3 antibody (data are from (22)), and (E) H3/Perth09 against 15 
$453 \mu \mathrm{g} / \mathrm{mL}$ Fl6v3 antibody. Relative resistance for WT is set as 1 . Mutants with a relative fitness of

454 less than 0.5 are excluded and shown as grey. Residues correspond to WT sequence are

455 boxed. (F) Fraction surviving for all single mutants across the HA protein are shown. Each data

456 point represents one mutant. Fraction surviving was computed as previously described (22).

457 Assuming no antibody-mediated enhancement of virus replication, the theoretical upper limit for

458 the fraction surviving is 1 , which indicates that the replication fitness is the same with and

459 without antibody selection (in practice, fraction surviving values slightly $>1$ can sometimes be

460 obtained due to the experimental error in qPCR and next-generation sequencing). Data for

461 H1/WSN against Fl6v3 were from a previous study (22). Data points that represent mutations at

462 residues 42 and 45 are colored in blue and red, respectively, and mutations at other residues

463 are in grey. (G) The crystal structures of H3 HA in complex with CR9114 (PDB 4FQY) (26) and

464 H5 HA in complex with CR9114 (PDB 4FQI) (26) were compared by aligning their CR9114

465 heavy chain variable domains. (H) Similarly, H3 HA in complex with FI6v3 (PDB 3ZTJ) (27) and

$466 \mathrm{H} 1 \mathrm{HA}$ in complex with FI6v3 (PDB 3ZTN) (27) were compared by aligning their heavy chain

467 variable domains. 
469 Table 1. Binding affinity of IgGs and Fabs against HAs of WT and different H3/HK68 HA2

470 mutants.

\begin{tabular}{|c|c|c|c|c|c|}
\hline $\mathbf{K}_{\mathbf{d}}$ in nM & WT & HA2-I45T & HA2-I45M & HA2-I45F & HA2-N49T \\
\hline CR9114 Fab & $43.2 \pm 0.4$ & $779.6 \pm 50.0$ & $319.1 \pm 12.1$ & n.b. & $11.3 \pm 0.4$ \\
\hline 27F3 Fab & $122.6 \pm 3.0$ & n.b. & n.b. & n.b. & $105.0 \pm 1.0$ \\
\hline CR9114 IgG & $<0.1$ & $164.8 \pm 2.3$ & $75.0 \pm 1.0$ & n.b. & $<0.1$ \\
\hline 27F3 IgG & $1.7 \pm 0.1$ & n.b. & n.b. & n.b. & $1.9 \pm 0.9$ \\
\hline FI6v3 IgG & $<0.1$ & $7.8 \pm 0.2$ & $6.7 \pm 0.1$ & $62.0 \pm 0.2$ & $<0.1$ \\
\hline S139/1 IgG & $2.1 \pm 0.1$ & $3.1 \pm 0.3$ & $1.9 \pm 0.1$ & $1.8 \pm 0.1$ & $2.8 \pm 0.1$ \\
\hline
\end{tabular}

n.b.: no binding 